\title{
Pseudomonas infection in chronic obstructive pulmonary disease
}
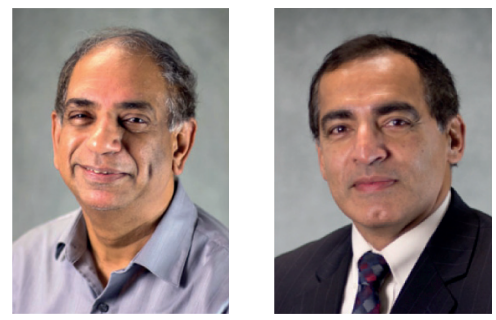

"...uncertainty exists regarding the

need for and approach to antibiotic

treatment of acute and chronic

$P$. aeruginosa infection in chronic

obstructive pulmonary disease."

\section{Ganapathi lyer Parameswaran ${ }^{\star 1,2}$ \& Sanjay Sethi ${ }^{2,3}$}

'Division of Infectious Diseases, Department of Medicine, University at Buffalo, State University of New York

Room 107, Research Building (20), VA Medical Center, 3495 Bailey Ave, Buffalo, NY 14215, USA

2VA Western New York Healthcare System, Buffalo, NY, USA

${ }^{3}$ Division of Pulmonary, Critical Care \& Sleep Medicine, Department of Medicine, University at Buffalo,

State University of New York, NY, USA

*Author for correspondence: Tel.: +1 7168628924 m gp36@buffalo.edu

Pseudomonas aeruginosa is a pulmonary pathogen in parenchymal infections such as ventilatorassociated pneumonia, and in airway infections such as cystic fibrosis (CF) and bronchiectasis. An unfolding story shows it to be a pathogen in chronic obstructive pulmonary disease (COPD) as well, with complex host-pathogen interactions.

Studies of $P$. aeruginosa in COPD are mostly cross-sectional in design. Sputum cultures and bronchoscopic sampling show that 3-20\% of stable COPD patients have $P$. aeruginosa in their lower airways; the frequency of colonization increases with worsening airflow obstruction [1,2]. Using bronchoscopy and protected specimen brush sampling, which avoids upper airway contamination, $P$. aeruginosa can be isolated from the lower airways of 3-10\% of COPD patients during an acute exacerbation [3]. P. aeruginosa is most likely to be present in patients with advanced COPD, especially during severe exacerbations requiring care in an intensive care unit (ICU) and mechanical ventilation [4]. Other risk factors for $P$. aeruginosa isolation at exacerbation are prior culture of $P$. aeruginosa, hospitalization and antibiotic use within the prior 3 months and stay in long-term nursing facilities [5].

Longitudinal studies have demonstrated that once established, Pseudomonas strains tend to persist for extended periods of time in CF patients. This contrasts with the findings in our longitudinal study in COPD. We examined 126 patients with COPD with monthly sputum cultures for up to 10 years [6]. P. aeruginosa was isolated one or more times in 39 patients (30\%), with 57 episodes of new-strain acquisition. Thirty-one of these strains were cleared from sputum after variable periods of time, 16 of which in less than a month. Persistent infection was noted with 13 out of the 57 strains, but the duration of infection with these
13 strains could not be accurately determined. Thus, unlike CF, acquisition of Pseudomonas does not always result in persistent infection in COPD. Application of molecular detection techniques has demonstrated that cultures are relatively insensitive in detecting bacterial pathogens in sputum. In our longitudinal study, when the same strain of $P$. aeruginosa was isolated intermittently from a patient, PCR analysis of sputum often revealed presence of $P$. aeruginosa, while cultures remained negative. From this we can conclude that estimates of $P$. aeruginosa carriage in COPD using sputum cultures alone underestimate its prevalence.

\section{"Sputum cultures and bronchoscopic sampling show that 3-20\% of stable chronic obstructive pulmonary disease patients have $P$. aeruginosa in their lower airways; the frequency of colonization increases with worsening airflow obstruction."}

When the prevalence of $P$. aeruginosa is examined in COPD by sputum cultures, no differences can be found between the stable state and during exacerbations. However, when strains of $P$. aeruginosa were characterized by molecular techniques to differentiate them into strains that were newly acquired at the time of exacerbation versus those that were pre-existing colonizing strains, a clear association between new strain acquisition and exacerbation emerged. In our longitudinal study, acquisition of a new strain of $P$. aeruginosa increased the relative risk for exacerbation by 3.36 ( $p<0.0001$ ), and 42.6\% of the new strain acquisitions were associated with an exacerbation [6]. New strain acquisitions

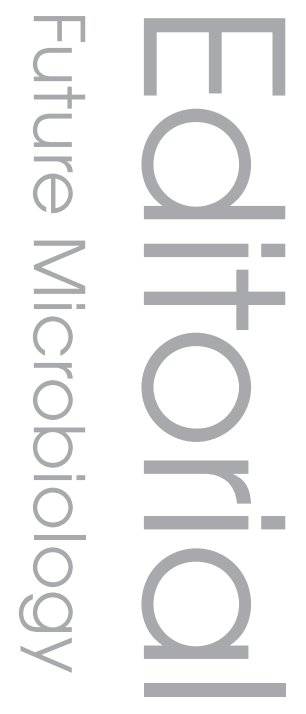


were also associated with production of serum antibodies directed specifically against the strain and enhanced airway and systemic inflammation, supporting a pathogenic role for this species in exacerbation [7].

\section{Outcomes \& course of $P$. aeruginosa acquisition in COPD}

In our experience, approximately $40 \%$ of new Pseudomonas strain acquisitions by COPD patients cause acute exacerbations; the other $60 \%$ of acquisitions result in colonization. In either circumstance (colonization or exacerbation), approximately $65 \%$ of the new strains are cleared after a variable period of time, most of which within a month. Approximately $35 \%$ of new strains persist in lower airways of COPD patients for prolonged periods of up to several months or years. The determinants of this hostpathogen relationship are still poorly understood. It is likely that differences in strain characteristics, such as mucoidy, biofilm formation and other virulence factors, as well as host characteristics, such as airflow obstruction and innate and adaptive immune responses, are important.

"Longitudinal studies of the
consequences of chronic Pseudomonas
infections in chronic obstructive
pulmonary disease are needed
to determine the appropriate
therapeutic approach."

Bacterial pathogen (including $P$. aeruginosa) acquisition and persistence in COPD that does not cause a change in baseline respiratory symptoms has been termed 'colonization' and presumed to be innocuous. Similar to observations in CF and bronchiectasis, several studies indicate that this colonization of the lower airways of COPD by pathogenic bacteria is associated with enhanced airway inflammation [8], and probably has deleterious consequences on the course of the disease [9].

\section{Biology of chronic Pseudomonas infection in COPD \& CF}

In $\mathrm{CF}$, once $P$. aeruginosa is established in the lower airways, it often persists for the lifespan of the patient unless eradication is carried out in very early stages of infection. Most often, a single clone is persistent in an individual patient. Chronic infection is characterized by mucoid phenotype, hypermutation with variability in phenotypes, resistance to multiple antibiotics, progressive decline in lung function and increase in mortality $[10,11]$.

Martinez-Solano et al. analyzed strains of P. aeruginosa from $13 \mathrm{COPD}$ patients [12]. Eight of these patients had multiple sequential episodes of $P$. aeruginosa infection. Strains from patients who had chronic infection showed increased frequency of mucoid change, hypermutation leading to the coexistence of multiple morphotypes within the same patient and increased antibiotic resistance over time. These strains produced fewer proteases, were less motile and were more prone to produce biofilms when compared with isolates of $P$. aeruginosa from patients with bloodstream infections. Similarly, strains from our longitudinal study that persisted were more likely to be or become mucoid, demonstrate microevolution of their accessory genome and demonstrate less cytotoxin (ExoU) production [13]. Furthermore, the genetic makeup of COPD strains compared with CF and ICU strains did not demonstrate disease-specific clones. Therefore, in contrast to $\mathrm{CF}$, persistence follows acquisition only in a minority of COPD patients. However, persistent $P$. aeruginosa strains in COPD behave in a manner similar to CF strains.

Unlike in CF, associations between acquisition of $P$. aeruginosa and deterioration of lung function, mortality and other adverse outcomes have not been established in COPD. Patients hospitalized for exacerbations with $P$. aeruginosa in respiratory cultures do have higher mortality rates compared with similar patients without $P$. aeruginosa [14]. Longitudinal studies of the consequences of chronic Pseudomonas infections in COPD are needed to determine the appropriate therapeutic approach.

Nearly $50 \%$ of COPD patients with moderate to severe disease, when examined by highresolution computerized tomography of the lungs, have bronchiectasis, especially in the lower lobes [15]. The frequency of bronchiectasis increases with worsening airflow obstruction. P. aeruginosa causes chronic infections and is associated with decline of lung function in patients with CF bronchiectasis and 'classic' non-CF bronchiectasis [16]. Therefore, it is an attractive hypothesis that there is a causative link between $P$. aeruginosa infection and the development of bronchiectasis in COPD. Studies to date suggest such a relationship; however, because of the small numbers of patients examined, a conclusive relationship has not been established. Martinez-Garcia et al. found bronchiectasis in $57.6 \%$ of 92 patients with moderate-to-severe COPD, and the presence of bronchiectasis correlated significantly with cultures of 
a pathogenic bacterial species from sputum [16]. Specifically, while six out of seven patients who had $P$. aeruginosa in sputum had bronchiectasis, the results did not reach statistical significance.

\section{"... it is an attractive hypothesis that there is a causative link between $P$. aeruginosa infection and the development of bronchiectasis in chronic obstructive pulmonary disease."}

Considerable uncertainty exists regarding the need for and approach to antibiotic treatment of acute and chronic $P$. aeruginosa infection in COPD. No randomized placebo-controlled trials exist that demonstrate whether specific antibiotic treatment of $P$. aeruginos $a$ changes outcomes in exacerbations of COPD. However, given its demonstrated pathogenic role and the severity of exacerbations in which it is isolated, several guidelines advise empiric treatment that covers $P$. aeruginosa in specific subgroups of patients. Since culture data are usually not available at the time of diagnosis of exacerbation, the clinician has to evaluate the likelihood that the patient has $P$. aeruginosa infection. The strongest predictor for $P$. aeruginosa infection is prior isolation of this species in sputum culture [5]. Other risk factors are severe exacerbations necessitating ICU admission and/or mechanical ventilation; hospitalization within the prior 3 months; recent broad-spectrum antibiotic therapy; residence in long-term nursing facilities; advanced COPD (Global Initiative for Obstructive Lung Disease [GOLD] stages III and IV); concomitant bronchiectasis; and chronic systemic corticosteroid treatment.

Antibiotic choice is limited in the out-patient setting, with ciprofloxacin as the only oral drug available. There are more choices for parenteral treatment in the in-patient setting. Benefits of using two antipseudomonal antibiotics in combination have not been demonstrated, and our preference is to use a single agent. In patients exposed to antibiotics in the previous 3 months, use of an alternative class of drug is prudent. Obtaining sputum cultures in these patients is also a good idea to confirm the presence of Pseudomonas and to guide antibiotic treatment. Well-designed trials are clearly needed to guide treatment of $P$. aeruginosa exacerbations.

Unlike in CF, whether chronic infection with $P$. aeruginosa in COPD needs specific treatment is uncertain. Small, uncontrolled trials using inhaled agents such as tobramycin and colistin have shown clinical and microbiological improvement in non-CF patients with chronic bronchial infections due to $P$. aeruginosa [17,18]. Several new developments in inhaled antibiotic therapy have made it feasible to deliver such treatment with ease to COPD patients; however, clinical evidence is lacking.

\section{Areas of uncertainty}

Several questions remain regarding Pseudomonas infection in COPD. Pathogen and host factors that determine the consequence of acquisition of Pseudomonas strains in COPD are poorly understood. What is unique about the subgroup of COPD patients in whom $P$. aeruginosa infection assumes features similar to CF? Studies examining the frequency of mutations and polymorphisms of the CFTR gene in COPD have yielded inconclusive or contradictory results $[19,20]$. Is there any benefit with inhaled antibiotics in reducing long-term decline in lung function in these patients? What is the optimal antibiotic regimen for $P$. aeruginosa exacerbations? Vaccines to protect against $P$. aeruginosa infections, using a variety of capsular and intracellular proteins, have been tested in mice and small human trials. Results have been inconclusive and no vaccine has been approved for clinical use.

\footnotetext{
Financial \& competing interests disclosure

The authors have no relevant affliations or financial involvement with any organization or entity with a financial interest in or financial conflict with the subject matter or materials discussed in the manuscript. This includes employment, consultancies, honoraria, stock ownership or options, expert testimony, grants or patents received or pending, or royalties.

No writing assistance was utilized in the production of this manuscript.
}

\section{References}

1. Marin A, Monso E, Garcia-Nunez M et al. Variability and effects of bronchial colonisation in patients with moderate COPD. Eur. Respir. J. 35(2), 295-302 (2010).

2. Engler K, Muhlemann K, Garzoni C, Pfahler H, Geiser T, Von Garnier C. Colonisation with Pseudomonas aeruginosa and antibiotic resistance patterns in COPD patients. Swiss Med. Wkly 142, W13509 (2012).

3. Soler N, Torres A, Ewig S et al. Bronchial microbial patterns in severe exacerbations of chronic obstructive pulmonary disease (COPD) requiring mechanical ventilation. Am. J. Respir. Crit. Care Med. 157(5 Pt 1), 1498-1505 (1998).
4. Rosell A, Monso E, Soler N et al. Microbiologic determinants of exacerbation in chronic obstructive pulmonary disease. Arch. Intern. Med. 165(8), 891-897 (2005).

5. Garcia-Vidal C, Almagro P, Romani V et al. Pseudomonas aeruginosa in patients hospitalised for COPD exacerbation: a prospective study. Eur. Respir. J. 34(5), 1072-1078 (2009). 
6. Murphy TF, Brauer AL, Eschberger K et al. Pseudomonas aeruginosa in chronic obstructive pulmonary disease. Am. J. Respir. Crit. Care Med. 177(8), 853-860 (2008).

7. Sethi S, Murphy TF. Infection in the pathogenesis and course of chronic obstructive pulmonary disease. N. Engl. J. Med. 359 (22), 2355-2365 (2008).

8. Sethi S, Maloney J, Grove L, Wrona C, Berenson CS. Airway inflammation and bronchial bacterial colonization in chronic obstructive pulmonary disease. Am. J. Respir. Crit. Care Med. 173(9), 991-998 (2006).

9. Banerjee D, Khair OA, Honeybourne D. Impact of sputum bacteria on airway inflammation and health status in clinical stable COPD. Eur. Respir. J. 23(5), 685-691 (2004).

10. Martin DW, Schurr MJ, Mudd MH, Govan JR, Holloway BW, Deretic V. Mechanism of conversion to mucoidy in Pseudomonas aeruginosa infecting cystic fibrosis patients. Proc. Natl Acad. Sci. USA 90 (18), 8377-8381 (1993).

11. Smith EE, Buckley DG, Wu Z et al. Genetic adaptation by Pseudomonas aeruginosa to the airways of cystic fibrosis patients. Proc. Natl Acad. Sci. USA 103(22), 8487-8492 (2006).

12. Martinez-Solano L, Macia MD, Fajardo A, Oliver A, Martinez JL. Chronic Pseudomonas aeruginosa infection in chronic obstructive pulmonary disease. Clin. Infect. Dis. 47(12), 1526-1533 (2008).

13. Rakhimova E, Wiehlmann L, Brauer AL, Sethi S, Murphy TF, Tummler B. Pseudomonas aeruginosa population biology in chronic obstructive pulmonary disease. J. Infect. Dis. 200(12), 1928-1935 (2009).

14. Montero M, Dominguez M, Orozco-Levi M, Salvado M, Knobel H. Mortality of COPD patients infected with multi-resistant Pseudomonas aeruginosa: a case and control study. Infection 37(1), 16-19 (2009).

15. Martinez-Garcia MA, Soler-Cataluna JJ, Donat Sanz Y et al. Factors associated with bronchiectasis in patients with COPD. Chest 140(5), 1130-1137 (2011).

16. Martinez-Garcia MA, Soler-Cataluna JJ, Perpina-Tordera M, Roman-Sanchez P, Soriano J. Factors associated with lung function decline in adult patients with stable non-cystic fibrosis bronchiectasis. Chest 132(5), 1565-1572 (2007).

17. Dal Negro R, Micheletto C, Tognella S, Visconti M, Turati C. Tobramycin nebulizer solution in severe COPD patients colonized with Pseudomonas aeruginosa: effects on bronchial inflammation. Adv. Ther. 25(10), 1019-1030 (2008).

18. Orriols R, Roig J, Ferrer J et al. Inhaled antibiotic therapy in non-cystic fibrosis patients with bronchiectasis and chronic bronchial infection by Pseudomonas aeruginosa. Respir. Med. 93(7), 476-480 (1999).

19. Artlich A, Boysen A, Bunge S, Entzian P, Schlaak M, Schwinger E. Common CFTR mutations are not likely to predispose to chronic bronchitis in northern Germany. Hum. Genet. 95(2), 226-228 (1995).

20. Tzetis M, Efthymiadou A, Strofalis S et al. CFTR gene mutations - including three novel nucleotide substitutions - and haplotype background in patients with asthma, disseminated bronchiectasis and chronic obstructive pulmonary disease. Hum. Genet. 108(3), 216-221 (2001). 